\title{
Untersuchung iiber die Haar- und Haargruppendichtigkeit an den Augenbrauen bei den Japanern.
}

\author{
von \\ Simoon Byon. \\ Aus dem Anatomischen Institut der Keio Universität, Tokyo.
}

\section{Einleitung.}

In der vorliegenden Arbeit verfolgte ich die Haar- und Haargruppenzahl an der Augenbraue in den verschiedenen Altersstufen bei den Japanern, und verglich mit denen an den anderen Kopfteilen.

\section{Material und Methode.}

Das mir zur Verfügung gestellte Material stammt aus 12 Feten von verschiedenen Embryonalmonaten und 14 Fällen von verschiedenen Altersstufen nach der Geburt.

Die Behandelungsweise wurde ganz nach voriger Mitteilung vorgenommen. Zur Berechnung der Haarzahl wurde die Haarbälge in 30 facher Vergrösserung auf ein Papierstück projiziert und gezeichnet, dann zählte ich die auf bestimmtes Quadrat $(100 \mathrm{qcm})$ dieses Papiers gezeichneten Haarbälge und Haarbalggruppen, wiederholte also diese Zählung in 10 verschiedenen Hautteilen der einzelnen Körperteile, addierte dann die gewonnene Zahl und rechnete den Mittelwert aus, den ich dann auf $1 \mathrm{qcm}$ Hautflächeninhalt umrechnete.

\section{Eigene Befunde.}

Die Haare sind einzeln oder zu Haargruppen implantiert. Nach meiner Untersuchung beläuft sich die Haarzahl einer Haargruppe auf 2-5 Haare. Jede Haargruppe ist von benachbarten Haargruppen durch haarfreie $\mathrm{Zwischenräume} \mathrm{getrennt.} \mathrm{Also} \mathrm{kann}$ man die Arten der Haargruppen unterscheiden, d. h. in zwei- bis fünftige Haargruppen. Zwecks besserer Erklärung soll das einzelne 
Haar als eine Haargruppe, d. h. einhaarige Haaryruppe, behandelt werden.

Im folgenden werde ich meine Befunde tabellarisch angeben (Tabelle 1).

\section{Zusammenfassung.}

Im folgenden werde zuerst die Haarzahl, dann die Haargruppenzahl, der Prozentsatz der verschiedenen Haargruppenarten und zuletzt die Vergleichung mit anderen Kopfteile besprochen.

1. Haarzahl auf $1 \mathrm{qcm}$ Hautflächeninhalt.

Wie in der Tabelle 1 gezeigt, ist die Haarzahl an der Augenbraue nach dem Alter verschieden gross. Beim Fetalleben, ist sie am grössten bei Fall 4 (6EM, 5061) und geringsten bei Fall 11 (10EM, 1601). Die vordere ist ungefähr 3 fach grösser als die letztere. Bei den Fällen nach der Geburt, zeigt Fall 13 (0.5M, 1629) die grösste Haarzahl, die fast gleich gross wie im 10EM ist. Aber die Haarzahl ist bei Fall 14 (1J3M, 951) ca. Hälfte und bei Fall 15 (6J, 636) ca. Drittel der Zahl im 5 Monate.

Im grossen und ganzen ist die Haarzahl am grössten im $6 \mathrm{Em}$ bryonalmonate, dann nimmt sie mit der Zunahme des Alters nach und nach ab. Bei den Erwachsenen weist sie keine erhebliche Schwankung auf, mit Ausnahme von Fall 25 (47J, 236), bei dem sie besonders klein ist.

2. Zahl der Haargruppen.

Ich kann 4 Arten von Haargruppen, die aus 1-5 Haaren bestehen, finden. Die gesamte Haargruppenzahl im $1 \mathrm{qcm}$ Hautflächeninhalt zeigt ungefähr die gleiche Schwankung nach verschiedenen Altersstufen wie die der Haarzahl. Sie ist am grössten im 5 Embryonalmonate (3110, durchschnittlich), von diesem Monate an, nimmt sie mit der Zunahme des Embryonalmonates nach und nach ab; sie ist am geringsten im $10 \mathrm{EM}$ (1125, durchschnittlich) bei Fetalleben. Bei den Fällen nach der Geburt hat. Fall 13 (0.5 M, 1017) auch die grösste Haargruppenzahl, folgt dann Fall 14 (1J3 M, 708). Am geringsten ist die Haarzahl bei Fall $25(47 \mathrm{~J}, 229)$. Bei den übrigen Fällen beträgt sie 344-528. 
3. Der Prozentsatz der einzelnen Haargruppenarten.

Der Prozentsatz der verschiedenen Haargruppenarten ist nach Altersstufen verschieden gross, und zeigt keine nennenswertige Altersveränderung wegen der individuellen Schwankung. Aber der Prozentsatz der verschiedenen Haargruppenarten zur gesamten Haargruppenzahl bei den einzelnen Individuen ist, ausnabmlos in jedem der untersuchten Fälle, immer am grössten bei der 1-haarigen Gruppe, dann folgen 2-haarige, 3-haarige und 4-haarige, und am geringsten bei der 5-haarigen. Nämlich, der Prozentsatz einer Haargruppe ist desto kleiner, je grösser die eine Haargruppe bildende Haarzahl ist.

Wenn man den durchschnittlichen Prozentsatz der verschiedenen Haargruppenarten bei den gesamten Feten mit demjenigen bei den Fällen nach der Geburt vergleicht, so findet man, dass bei den Feten der Prozentsatz der 1-haarigen und 2-haarigen Gruppe grösser, jedoch der der 3-haarigen, 4- haarigen und 5-haarigen kleiner als der bei den Fällen nach der Geburt.

4. Vergleich der Befunde an der Augenbraue mit denen an den anderen Kopfteilen.

Wenn man die obigen Befunde an der Augenbraue mit denen an dem Scheitel und der Stirn (bei den japanischen Feten von $\mathrm{K}$ ato und bei den Japanern nach der Geburt von $T$ an i g u chi et $\mathrm{S}$ h i b a y a m a untersucht) vergleicht, so kann man ersehen, dass die Haar- und Haargruppenzahl an der Augenbraue verhältnismässig grösser als an dem Scheitel und der Stirn in jeden untersuchten Altersstufen sind, aber doch sie die gleichartige Schwankung nach den Altersstufen wie an dem Scheitel und der Stirn zeigen.

Von $\mathrm{K}$ a to sind 8 Haargruppenarten am Scheitel bei den japanischen Feten gefunden, und von $\mathrm{T}$ a $\mathrm{ni} \mathrm{g} \mathrm{u} \mathrm{ch}$ i et $\mathrm{Sch}$ i ba y a ma 9 Haargruppenarten am Scheitel bei Japanern nach der Geburt beschrieben, jedoch unterscheide ich an der Augenbraue nur 5 Arten.

Bezüglich des Prozentsatzes der verschiedenen Haargruppenarten bei einzelnen Individuen, ist der durchschnittliche prozentuale Wert am Scheitel bei den Feten von $\mathrm{K}$ a t 0 , am höchsten bei der 2-haarigen Haargruppe (37.4\%), dann folgen 3-haarige, 4-haarige, 5-haarige und 1-haarige (7.7\%), obwohl der Wert an den Augenbrauen bei 1-haarigen am höchsten $(65.7 \%)$ ist.

Bei den Fällen nach der Geburt ist er am Scheitel von $\mathrm{T}$ a $\mathrm{n}$ i$\mathrm{g} \mathrm{u} \mathrm{chi}$ und $\mathrm{Sh}$ i ba y a ma am höchsten bei der 3-haarigen Gruppe 


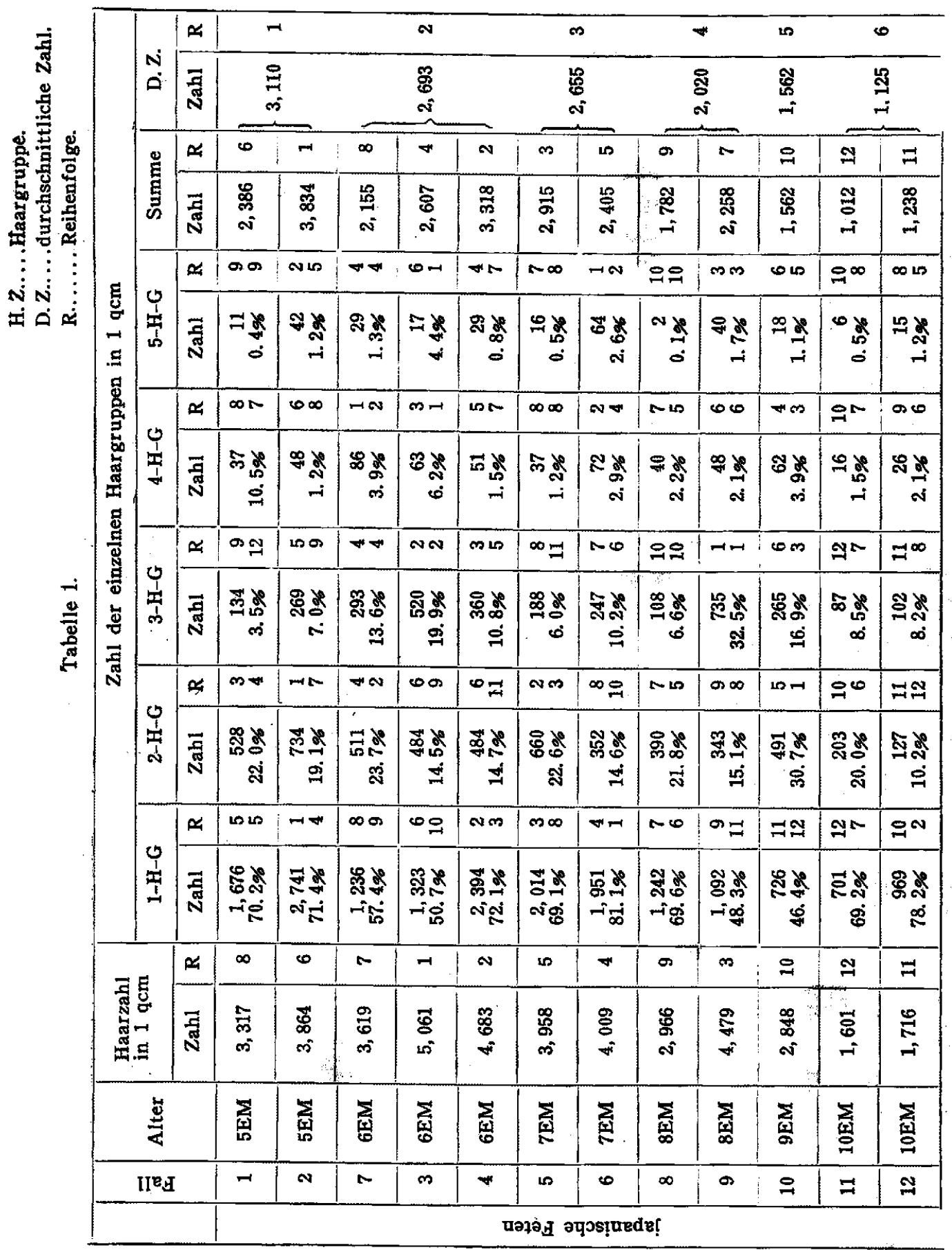




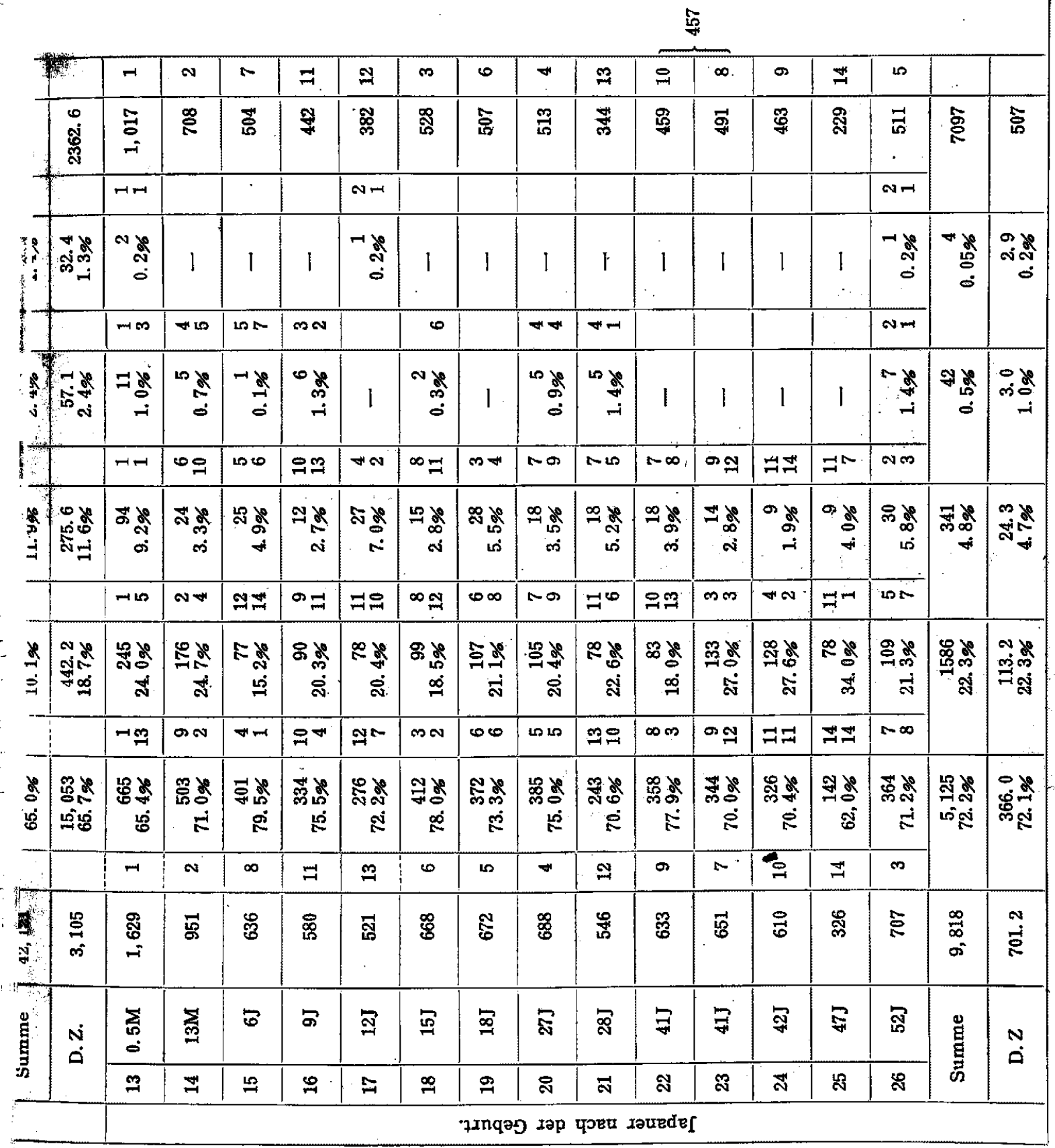


(35.5\%), dann folgen 2-haarige, 4-haarige, 1-haarige (13.3\%) und 5haarige. Aber hat die Augenbraue bei der 1-haarigen (72.1\%) den grössten Prozentsatz. Obwohl der Prozentsatz der verschiedenen Haargruppenzahl zur gesamten Haargruppenzahl am Scheitel nach verschiedenen Altersstufen schwankt, bleibt doch die Reihenfolge der Grösse des Prozentsatzes an der Augenbraue immer unverändert. Das heisst, er ist bei der 1-haarigen Gruppe am grössten, dann folgen 2-haarige, 3-haarige und 4-haarige; or ist am geringsten bei der 5-haarigen.

\section{Literatur.}

1) Goto, K., Über die Haarzahl und Bestimmungsmethode des Oberfächeninhaltes der behaarten Kopfhautteile. Tokyo-Iji-Shinshi, Jahrg. 1931 (japanisch).

2) J a das sohn, J., Handbuch der Haut- und Geschlechtskrankheiten. Bd. 1/1. Anatomie der Haut. 1927, Berlin.

3) Miki, N., Pri la distribuo de haroji sur la vizago. Tokyo Igakkai-Zasshi, Bd. 48, 1938 (japanisch).

4) Tan i g u hi, T., Über die Verteilung der Schweissdrüsen beim Menschen. Nagoya-Iji-Shinshi, Bd. 8, 1934 (japanisch).

5) T a n ig u chi, T. und $\mathrm{Sh}$ i b a y a $\mathrm{ma}, \mathrm{H}$., Die Dichtigkeit der Körperbehaarung bei einigen Rassen. Fol. anat. jap., Bd. 13, 1935.

6) Taniguchi, T., Quantitative Untersuchung der Anhangsorgane der Haut bei einem Hydrokephalus. Fol. anat. jap., Bd. 13, 1935.

7) Taniguchi, T. und $S$ hibay a na, H., Die Dichtigkeit der Körperbehaarung bei den Japanern. Fol. anat. jap., Bd. 13, 1935.

8) Ka to, S., Untersuchungen über die Haar- und Haargruppendichtigkeit bei den japanischen Feten. Mit II Textfiguren. Fol. anat. jap., Bd. 14, 1936.

9) Berger, E., Beiträge zur Morphologie der behaarten Kopfhaut und der Aug. enbrauen. Über eine Haarbrücke zwischen der behaarten Kopfhaut und den Augenbrauen. Maph Jahrb., Bd. 48, 1914. 